\title{
THE LINDELÖF DEGREE OF SCATTERED SPACES AND THEIR PRODUCTS
}

\author{
MARLENE E. GEWAND \\ (Received 1 December 1982, revised 22 March 1983) \\ Communicated by J. H. Rubinstein
}

\begin{abstract}
Different methods are used to show that a finite or countable product of Lindelö scattered spaces is Lindelöf. Also, a technique of Kunen is modified to yield results concerning the Lindelöf degree of the $G_{\delta^{-}}$and $G_{\alpha}$-topologies on the countable product of compact scattered spaces.

1980 Mathematics subject classification (Amer. Math. Soc.): primary 54 D 20; secondary 54 B 10. Key wyords and phrases: Lindelöf degree, scattered spaces, product, $G_{\boldsymbol{\delta}^{-}}$-topology, compact, totally Lindelöf, $\mathscr{P}$-space.
\end{abstract}

\section{Introduction}

Since 1947, when R. H. Sorgenfrey [7] gave an example of a Lindelöf space whose cartesian product with itself was not normal, numerous questions have arisen concerning the products of Lindelöf spaces. We examine here the products of Lindelöf scattered spaces. Telgársky [9] has shown that the product of a Lindelöf $C$-scattered space with a Lindelöf space is Lindelöf. We show by a different method that the finite product of Lindelöf scattered spaces is Lindelöf. By looking at $\mathscr{P}$-spaces and also by examining the totally Lindelöf property, we are able to show that a countable product of Lindelöf scattered spaces is Lindelöf.

In Section 3 we look at the Lindelöf degree of the $G_{\delta^{-}}$and $G_{\alpha^{-}}$-topologies on countable products of compact scattered or Lindelöf scattered spaces. A technique of Kunen [3] is modified to yield some results here.

This work was partially supported by a grant from the Naval Academy Research Council.

(C) 1984 Australian Mathematical Society 0263-6115/84 \$A2.00+0.00 
All spaces are assumed to be Hausdorff regular. A space $X$ is said to be scattered if every non-empty subspace of $X$ contains an isolated point. All ordinal spaces are scattered. Given a space $X, X_{\delta}$ will represent the set $X$ with the topology generated by the $G_{\delta}$-sets of $X$. Similarly, $X_{\alpha}$ will denote the set $X$ with the topology generated by the $G_{\alpha}$-sets (sets which are the intersection of no more than $\alpha$ open sets) of $X . L(X)$ denotes the Lindelöf degree of $X$ (see Juhász [2]). $|X|$ denotes the cardinality of $X$. The notation $p_{n}(Y)$ is used for the $n$th projection of $Y$, a subset of a product space.

\section{Finite and countable products}

As indicated by the following lemma, the $G_{\delta}$-topology on a space $X$ may be useful in determining the Lindelöf degree of the product of $X$ with another space.

LEMma 2.1. If $L\left(X_{\delta}\right) \leqslant \beta$, then $L(X \times Y) \leqslant \beta$ for every Lindelöf space $Y$.

Proof. Let $\mathcal{C}$ be an open cover of $X \times Y$. Without loss of generality, we may assume that every member of $\mathcal{C}$ is of the form $G \times H$ where $G$ and $H$ are open in $X$ and $Y$, respectively.

For each $x \in X,\{x\} \times Y$ is Lindelöf and hence it can be covered by a countable subfamily $\mathcal{C}_{x} \subseteq \mathcal{C}$. For each $x \in X$, let $G_{x}=\cap\left\{G: G \times H \in \mathcal{C}_{x}\right\}$. Since each $G_{x}$ is a $G_{\delta}$-set and $L\left(X_{\delta}\right) \leqslant \beta$, there is a subfamily $\left\{G_{x(\gamma)}: \gamma \leqslant \beta\right\}$ of $\left\{G_{x}: x \in X\right\}$ which covers $X$. Then $\cup\left\{\mathcal{C}_{x(\gamma)}: \gamma \leqslant \beta\right\}$ is a subfamily of $\mathcal{C}$ of cardinality no greater than $\beta$ which covers $X \times Y$.

Of course this result can easily be generalized to higher cardinalities:

$$
\text { If } L\left(X_{\alpha}\right) \leqslant \beta \text { and } L(Y) \leqslant \alpha \text {, then } L(X \times Y) \leqslant \alpha \cdot \beta \text {. }
$$

But of interest to us here is the countable case as stated in Lemma 2.1.

P. Meyer [4] showed that a compact space $X$ is scattered if and only if $X_{\delta}$ is Lindelöf. We give a simple proof of a strengthening in one direction of this result without the Cantor-Bendixon decomposition type argument of Meyer.

THEOREM 2.2. If $X$ is scattered and $L(X)=\omega$, then $L\left(X_{\delta}\right)=\omega$.

Proof. Let $\mathcal{C}$ be a cover of $X$ by $G_{\delta}$-sets. Let $U=\{x \in X: x \in H$ and $H$ open in $X$ implies $H$ cannot be covered by a countable subfamily of $\mathcal{C}\}$. $U$ is closed.

Suppose $U \neq \varnothing$. Then $U$ has an isolated point $x$ and there is an open set $G \subseteq X$ such that $G \cap U=\{x\}$. Choose $C(x) \in \mathcal{C}$ such that $x \in C(x)$. We may 
assume, without loss of generality, that $C(x)=\cap\{G(n): n<\omega\}$ where, for each $n<\omega, G(n)$ is open and $G(n+1) \subseteq \overline{G(n+1)} \subseteq G(n) \subseteq G$. We consider $\overline{G(n)}$ $-G(n+1)$ for each $n<\omega$. Each $y \in(\overline{G(n)}-G(n+1)) \subseteq X-U$ has a

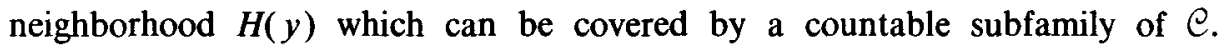
Furthermore, the family $\{H(y): y \in \overline{G(n)}-G(n+1)\}$ has a countable subcover since $L(\overline{G(n)}-G(n+1))=\omega$. Hence each $\overline{G(n)}-G(n+1)$ can be covered by a countable subfamily $\mathcal{C}(n) \subseteq \mathcal{C}$. Then $\{C: C \in \mathcal{C}(n), n<\omega\} \cup$ $\{C(x)\}$ is a countable subfamily of $\mathcal{C}$ covering $G$, which contradicts $x \in U$. Thus $U=\varnothing$.

Since $U=\varnothing$, there is a neighborhood $H(x)$ of $x$, for each $x \in X$, such that $H(x)$ can be covered by a countable subfamily of $\mathcal{C}$. $X$ is Lindelöf, so $\{H(x)$ : $x \in X\}$ can be reduced to a countable subcover which in turn yields a countable subcover of $\mathcal{C}$.

An extensive study of covering properties of $C$-scattered spaces was made by Telgársky [8], [9]. A space $X$ is said to be $C$-scattered if every non-empty closed subspace has a point with a compact neighborhood in that subspace. It was shown by Telgársky [9] that the product of a Lindelöf $C$-scattered space with a Lindelöf space is Lindelöf. By Lemma 2.1 and Theorem 2.2 we have the following corollaries.

Corollary 2.3. If $L(X)=L(Y)=\omega$ and $X$ is scattered, then $L(X \times Y)=\omega$.

COROLlary 2.4. A finite product of Lindelöf scattered spaces is Lindelöf.

COROLlaRY 2.5. If $X$ is Lindelöf and scattered and if each point of $X$ is $a G_{\delta}$, then $|X|<\omega$.

A space $X$ is a $\mathcal{P}$-space if every $G_{\delta}$-set in $X$ is open. Combining N. Noble's [5] results on $\mathcal{P}$-spaces with Theorem 2.2 , we can give a simple proof that the countable product of Lindelöf scattered spaces is Lindelöf.

THEOREM 2.6. [5] A countable product of Lindelöf $\mathcal{P}_{\text {-spaces is Lindelöf. }}$

COROLLARY 2.7. A countable product of Lindelöf scattered spaces is Lindelöf.

Proof. Let $\{X(n): n<\omega\}$ be a family of Lindelöf scattered spaces. Then by Theorem 2.2, each $(X(n))_{\delta}$ is a Lindelöf $\mathcal{P}$-space. $\Pi_{n<\omega}(X(n))_{\delta}$ is Lindelöf by Theorem 2.6 and since $\Pi_{n<\omega}(X(n))_{\delta}$ maps continuously onto $\Pi_{n<\omega} X(n)$, $\Pi_{n<\omega} X(n)$ is also Lindelöf. 
Another method of determining the Lindelöf degree of a countable product of Lindelöf scattered spaces is by means of totally Lindelöf spaces. J. E. Vaughan has examined this property and its related properties in several papers; [10] and [11] are primary sources. There are spaces which are Lindelöf but not totally Lindelöf [10]. We begin with some definitions.

A filter base $\mathcal{G}$ is said to be finer than the filter base $\mathscr{F}$ if every member of $\mathscr{F}$ contains a member of $\mathcal{G}$. A filter base is said to be total [10] if each finer filter base has an adherent point (that is, each finer filter base clusters).

A space $X$ is totally Lindelö [11] if given a filter base $\mathscr{F}$ on $X$ which is stable under countable intersections (that is, if $F(n) \in \mathcal{F}$ for all $n<\omega$, then there exists $F \in \mathscr{F}$ such that $F \subseteq \cap\{F(n) ; n<\omega\})$, there is a filter base $\mathcal{G}$ on $X$ such that

(i) $\mathcal{G}$ is stable under countable intersections,

(ii) $\mathcal{G}$ is finer than $\mathscr{F}$, and

(iii) $\mathcal{G}$ is total.

With the following lemma we will be able to establish a relationship between Lindelöf scattered spaces and totally Lindelöf spaces.

LEMMA 2.8. If $X$ is the union of a countable number of subsets each of which is totally Lindelöf, then $X$ is totally Lindelöf.

Proof. Let $X=\bigcap\{A(n): n<\omega\}$ where each $A(n)$ is totally Lindelöf. Let $\mathscr{F}$ be a filter base on $X$ which is stable under countable intersections.

For each $n<\omega$, we define a family $\mathscr{F}(n)$ as follows: if there exists $F \in \mathscr{F}$ for which $F \cap A(n)=\varnothing$, let $\mathscr{F}(n)=\varnothing$; otherwise let $\mathscr{F}(n)=\{F \cap A(n): F \in \mathscr{F}\}$.

We observe that there exists $n^{*}<\omega$ for which $\mathscr{F}\left(n^{*}\right) \neq \varnothing$. If this were not the case, then for each $n<\omega$, we could choose $F(n) \in \mathscr{F}$ for which $F(n) \cap A(n)=\varnothing$. Since $\mathcal{F}$ is stable under countable intersections, there exists $G \subseteq \cap\{F(n) ; n<\omega\}$ and since $X=\cup\{A(n): n<\omega\}$, there exists $m<\omega$ such that $G \cap A(m) \neq \varnothing$. But $(G \cap A(m)) \subseteq(F(m) \cap A(m))=\varnothing$ yields a contradiction.

To see that $\mathscr{F}\left(n^{*}\right)$ is stable under countable intersections, let $\{F(n): n<\omega\}$ be a countable subfamily of $\mathscr{F}\left(n^{*}\right)$. For each $n<\omega$, there exists $G(n) \in \mathscr{F}$ such that $F(n)=G(n) \cap A\left(n^{*}\right)$. Since $\mathscr{F}$ is stable there exists $G \in \mathscr{F}$ such that $G \subseteq$ $\cap\{G(n): n<\omega\}$. Now $G \cap A\left(n^{*}\right) \neq \varnothing$ and $\left(G \cap A\left(n^{*}\right)\right) \subseteq \cap\left\{G(n) \cap A\left(n^{*}\right)\right.$ : $n<\omega\}=\bigcap\{F(n): n<\omega\}$.

Since $\mathscr{F}\left(n^{*}\right)$ is stable and $A\left(n^{*}\right)$ is totally Lindelöf, $\mathscr{F}\left(n^{*}\right)$ has a finer filter base $\mathcal{G}\left(n^{*}\right)$ which is total and stable under countable intersections. We note that $\mathcal{G}\left(n^{*}\right)$ is finer than $\mathscr{F}$ and thus $X$ is totally Lindelöf.

THEOREM 2.9. If $X$ is Lindelöf and scattered, then $X$ is totally Lindelöf. 
Proof. Suppose $X$ is Lindelöf and scattered. Let $A=\{x \in X$ : every neighborhood of $x$ fails to be totally Lindelöf $\}$.

If $X=\varnothing$, we are finished because for each $x \in X$, there is a neighborhood $N(x)$ which is totally Lindelöf. The family $\{N(x): x \in X\}$ can be reduced to a countable subcover of $X$ and Lemma 2.8 can be applied.

If $A \neq \varnothing$, then $A$ has an isolated point $a$ and there exists an open set $G \subseteq X$ such that $G \cap A=\{a\}$. We may assume, without loss of generality, that every point of $X$ except $a$ has a neighborhood which is totally Lindelöf. Suppose $\mathscr{F}$ is a filter base on $X$ which is stable under countable intersections and suppose some finer filter base $\mathcal{G}$, which is stable under countable intersections, does not cluster at $a$. Then there exists $G \in \mathcal{G}$ such that $a \notin \bar{G}$. If we can show that $\bar{G}$ is totally Lindelöf, then $\mathcal{G}$ will cluster in $\bar{G}$ and we will be finished. For each $x \in \bar{G}$, there is a neighborhood $N(x)$ which is totally Lindelöf. Now $\{N(x): x \in X\}$ is an open cover of $\bar{G}$ which is Lindelöf. Hence $\bar{G}$ is a countable union of subsets each of which is totally Lindelöf and by Lemma $2.8, \bar{G}$ is totally Lindelöf.

With Theorem 2.9 and the following theorem of Vaughan, we are able to reach our conclusion about countable products of Lindelöf scattered spaces in Theorem 2.11 .

THEOREM 2.10. [11] A countable product of totally Lindelöf spaces is Lindelöf.

THEOREM 2.11. A countable product of Lindelöf scattered spaces is Lindelöf.

\section{The $G_{\delta^{-}}$and $G_{\alpha^{-}}$-topologies}

K. Kunen [3] has shown with a most beautiful technique that the Lindelöf degree of the box product of a countable number of compact scattered spaces is no greater than $c$, the cardinality of the continuum. This technique is modified to reach conclusions about the cartesian product.

The Cantor-Bendixon decomposition of a space $X$ is a non-increasing sequence of closed sets of $X$ defined inductively as follows: Let

$X^{(0)}=X$,

$X^{(\alpha+1)}=\left\{x \in X^{(\alpha)}: x\right.$ is not isolated in $\left.X^{(\alpha)}\right\}$, and

$X^{(\lambda)}=\cap\left\{X^{(\alpha)}: \alpha<\lambda\right\}$ for $\lambda$ a limit ordinal.

$X$ is scattered if and only if there exists an $\alpha$ such that $X^{(\alpha)}=\varnothing$. If $X$ is scattered and compact, then the first $\alpha$ for which $X^{(\alpha)}=\varnothing$ is a successor ordinal $\alpha=\beta+1$ and $X^{(\beta)}$ is finite. In this case, we say the rank of $X$ is $\beta$. 
THEOREM 3.1. If $X(n)$ is compact and scattered for each $n<\omega$, then $L\left(\left(\Pi_{n<\omega} X(n)\right)_{\delta}\right) \leqslant c$.

Proof. Let $\mathcal{C}$ be a cover of $\Pi_{n<\omega} X(n)$ by $G_{\delta}$-sets. Without loss of generality, we may assume $\mathcal{C}$ to be a closed cover and if $C \in \mathcal{C}$, then $C=\cap\left\{G(C)_{i}: i<\omega\right\}$ with $G(C)_{i}$ open in $\prod_{n<\omega} X(n)$ for each $i<\omega$.

Consider the tree $T=\cup\left\{c^{\gamma}: \gamma<\omega_{1}\right\}$. For $t \in T$, denote the domain of $t$ by $\operatorname{dom}(t)$ and for $\xi<c$, let $t \xi$ be the extension of $t$ where $t \xi(\operatorname{dom}(t)+1)=\xi$. $t \uparrow \gamma$ will denote the restriction of $t$ to $\gamma$ and 0 is the empty function $(\operatorname{dom}(0)=\varnothing)$.

We will define, by induction on $\operatorname{dom}(t)$, closed Kunen sets $K(t)$ in such a way that a subfamily of $\{K(t): t \in T\}$ refines $\mathcal{C}$ and since $|T|=c$, we will be finished. Our sets $K(t)$ will be required to satisfy conditions similar to those in Kunen's theorem, namely:

(i) $K(0)=\Pi_{n<\omega} X(n)$, and for each $t \in T$,

(ii) $K(t) \subseteq \cup\{K(t \xi): \xi<c\}$,

(iii) $K(t)=\bigcap\{K(t \mid \gamma): \gamma<\operatorname{dom}(t)\}$ if $\operatorname{dom}(t)$ is a limit ordinal, and

(iv) for each $\xi<c$, either there exists $C \in \mathcal{C}$ such that $K(t \xi) \subseteq C$ or there exists $n<\omega$ for which rank $p_{n}(K(t \xi))<$ rank $p_{n}(K(t))$.

If these conditions are met, then we will have our refinement. The argument is like Kunen's. If $x \in \Pi_{n<\omega} X(n)$, then by (i), (ii), and (iii), there is a function $t \in T$ such that $x \in K(t \uparrow \gamma)$ for every $\gamma<\omega_{1}$. The ranks of $p_{n}(K(t \uparrow \gamma))$, for each $n$, are non-increasing and thus eventually constant. So by (iv), we must eventually get inside a covering set (that is, inside a member of the cover $\mathcal{C}$ ).

Our modification of the Kunen technique comes in the way we define our Kunen sets, $K(t)$. We define $K(0)=\Pi_{n<\omega} X(n)$ and we take intersections at the limit stages. Now suppose $K(t)$ has already been defined; we will define $K(t \xi)$ for each $\xi<c$. We let $\beta_{n}=$ rank $p_{n}(K(t))$ and $Z(n)=\left(p_{n}(K(t))\right)^{\left(\beta_{n}\right)}$. Since each $Z(n)$ is finite, there exists a subfamily $\mathcal{C}^{\prime} \subseteq \mathcal{C}$ of cardinality $c$ such that $\mathcal{C}^{\prime}$ covers $\Pi_{n<\omega} Z(n)$. Let $\mathcal{S}=\left\{G\right.$ : $G$ is of the form $G=G(C)_{i}$ for some $\left.C \in \mathcal{C}^{\prime}, i<\omega\right\}$. The sets $K(t \xi), \xi<c$, will list the $c$ sets $K$ such that either (a) $K=C \cap K(t)$ for some $C \in \mathcal{C}^{\prime}$ or (b) $K$ is a box, where for some $n$,

(1) $p_{n}(K)=p_{n}(K(t))-\cup\left\{p_{n}(G): G \in \mathcal{G}^{\prime}\right\}$ where $\mathcal{G}^{\prime} \subseteq \mathcal{G}$ is finite and $Z(n) \subseteq$ $\cup\left\{p_{n}(G): G \in \mathcal{G}^{\prime}\right\}$, and

(2) $p_{m}(K)=p_{m}(K(t))$ for each $m \neq n$.

Conditions (i) and (iii) are obviously met; condition (iv) will be satisfied because of (b) (1) of the definition. We show that condition (ii) is met by assuming $x \in K(t)$ and $x \notin K(t \xi)$ of type (b). Then for each $n<\omega$ and for each finite subfamily $\mathcal{G}^{\prime} \subseteq \mathcal{G}, Z(n) \subseteq \cup\left\{p_{n}(G): G \in \mathcal{G}^{\prime}\right\}$ implies $x(n)=p_{n}(x) \in$ $\cup\left\{p_{n}(G): G \in \mathcal{G}^{\prime}\right\}$. Furthermore, there exists $z(n) \in Z(n)$ such that for every 
$G \in \mathcal{G}, z(n) \in p_{n}(G)$ implies $x(n) \in p_{n}(G)$. So if $C \in \mathcal{C}^{\prime}$ and if $z(n) \in p_{n}(C)=$ $p_{n}\left(\cap\left\{G(C)_{i}: i<\omega\right\}\right)=\cap\left\{p_{n}\left(G(C)_{i}\right): i<\omega\right\}$, then $x(n) \in p_{n}(C)$. Defining $z \in$ $\Pi_{n<\omega} Z(n)$ so that $p_{n}(z)=z(n)$ for each $n<\omega$, we have for each $C \in \mathcal{C}^{\prime}, z \in C$ implies $x \in C$. Thus choosing $C \in \mathcal{C}^{\prime}$ such that $z \in C$, we have $x \in C \cap K(t)$, a Kunen set of type (a).

The following corollary easily follows from Theorem 3.1.

COROLlaRY 3.2. If $X(n)$ is a $\sigma$-compact, scattered space for each $n<\omega$, then $L\left(\left(\Pi_{n<\omega} X(n)\right)_{\delta}\right) \leqslant c$.

With appropriate changes in the proof of Theorem 3.1, we may further extend the result.

THEOREM 3.3. Under $G C H$, if $X(n)$ is compact and scattered for each $n<\omega$ and if $\alpha$ is a limit cardinal with $\operatorname{cf}(\alpha)>\omega$, then $L\left(\left(\Pi_{n<\omega} X(n)\right)_{\alpha}\right) \leqslant \alpha$.

Given the closed cover $\mathcal{C}$ of $I_{n<\omega} X(n)$ by $G_{\alpha}$-sets, if $C \in \mathcal{C}$, then $C=$ $\cap\left\{G(C)_{\beta}: \beta<\alpha\right\}$ with $G(C)_{\beta}$ open in $\Pi_{n<\omega} X(n)$ for each $\beta<\alpha$. The proof requires using the tree $T=\bigcup\left\{\alpha^{\gamma}: \gamma<\omega_{1}\right\}$ and the Kunen sets $K(t)$ are defined, by induction on $\operatorname{dom}(t)$ to meet the conditions (i)-(iv) of the proof of Theorem 3.1. To define the sets $K(t \xi)$, for $\xi<\alpha$, we follow the route of that proof, but use the family $\mathcal{C}=\left\{G\right.$ : $G$ is of the form $G=G(C)_{\beta}$ for some $\left.C \in \mathcal{C}^{\prime}, \beta<\alpha\right\}$.

We turn our attention now to Lindelöf scattered spaces. It is known that if $X$ is a scattered Lindelö space and $\alpha$ is the first ordinal such that $X^{(\alpha)}=\varnothing$, then either

(a) $\operatorname{cf}(\alpha)=\omega$, or

(b) $\alpha$ is a successor ordinal $\beta+1$ and $\left|X^{(\beta)}\right| \leqslant \omega$.

If condition (b) is met, we may still call $\beta$ the rank of $X$.

There are spaces which satisfy condition (a) but which are not Lindelöf. For example, if $X=\omega_{1} \dot{\cup} \omega_{\omega}$ (the disjoint union), then $\alpha=\omega_{\omega}$ and $X$ is not Lindelöf.

Question: If $X(n)$ is Lindelof and scattered for each $n<\omega$, then what can be said about $L\left(\left(\Pi_{n<\omega} X(n)\right)_{\delta}\right)$ ?

To answer this question, it may be necessary to consider several cases depending upon the type of Cantor-Bendixon decomposition each space $X(n)$ possesses.

\section{References}

[1] R. Engelking, General topology (Polish Scientific Publishers, Warsaw, 1977).

[2] I. Juhász, Cardinal functions in topology (Math. Centre Tracts 34, Mathematical Centre, Amsterdam, 1971). 
[3] K. Kunen, 'Paracompactness of box products of compact spaces', Trans. Amer. Math. Soc. 240 (1978), 307-316.

[4] P. R. Meyer, 'Function spaces and the Aleksandrov-Urysohn conjecture', Ann. Mat. Pura Appl. 86 (1970), 25-29.

[5] N. Noble, 'Products with closed projections II', Trans. Amer. Math. Soc. 160 (1971), 169-183.

[6] M. E. Rudin, Lectures on set theoretic topology (CBMS Regional Conference Series in Mathematics 23, Amer. Math. Soc., Providence, R. I., 1975).

[7] R. H. Sorgenfrey, 'On the topological product of paracompact spaces', Bull. Amer. Math. Soc. 53 (1947), 631-632.

[8] R. Telgársky, 'C-scattered and paracompact spaces', Fund. Math. 73 (1971), 59-74.

[9] R. Telgársky, 'Concerning the product of paracompact spaces', Fund. Math. 74 (1972), 153-159.

[10] J. E. Vaughan, Total nets and filters, Topology Proc. Memphis State Univ. Conf., pp. 259-265 (Marcel Dekker Lecture Notes 24, 1976).

[11] J. E. Vaughan, 'Products of topological spaces', General Topology and Appl. 8 (1978), 207-217.

Lockheed Missiles and Space Co., Inc.

1111 Lockheed Way

$0 / 81-15, \mathrm{~B} / 157$

Sunnyvale, California 94086

U.S.A. 